\title{
Forced Crowding of colloids by thermophoresis and convection in a custom liquid Clusius-Dickel micro- device
}

\author{
Brandon S. Light ${ }^{1}$, Miguel Zepeda-Rosales ${ }^{2}$, Youli Li $^{2 *}$, Cyrus R. Safinya ${ }^{3 *}$ \\ ${ }^{1}$ Materials Department, Chemical Engineering Department, University of California, Santa \\ Barbara, California 93106, USA \\ ${ }^{2}$ Materials Research Laboratory, University of California, Santa Barbara, California 93106, USA \\ ${ }^{3}$ Materials Department, Physics Department, Molecular, Cellular, and Developmental Biology \\ Department, University of California, Santa Barbara, California 93106, USA
}

*Corresponding author contact information: youli@mrl.ucsb.edu, cyrussafinya@ucsb.edu

\section{SUPPLEMENTARY INFORMATION}

Experimental Setup. Figure S1 is a close-up photograph of the Clusius-Dickel micro-device used in experiments annotated with labels for relevant components. Figure S2 shows the entire experimental apparatus, including the micro-device, its auxiliary temperature control components, and the optical microscope used to obtain data. 


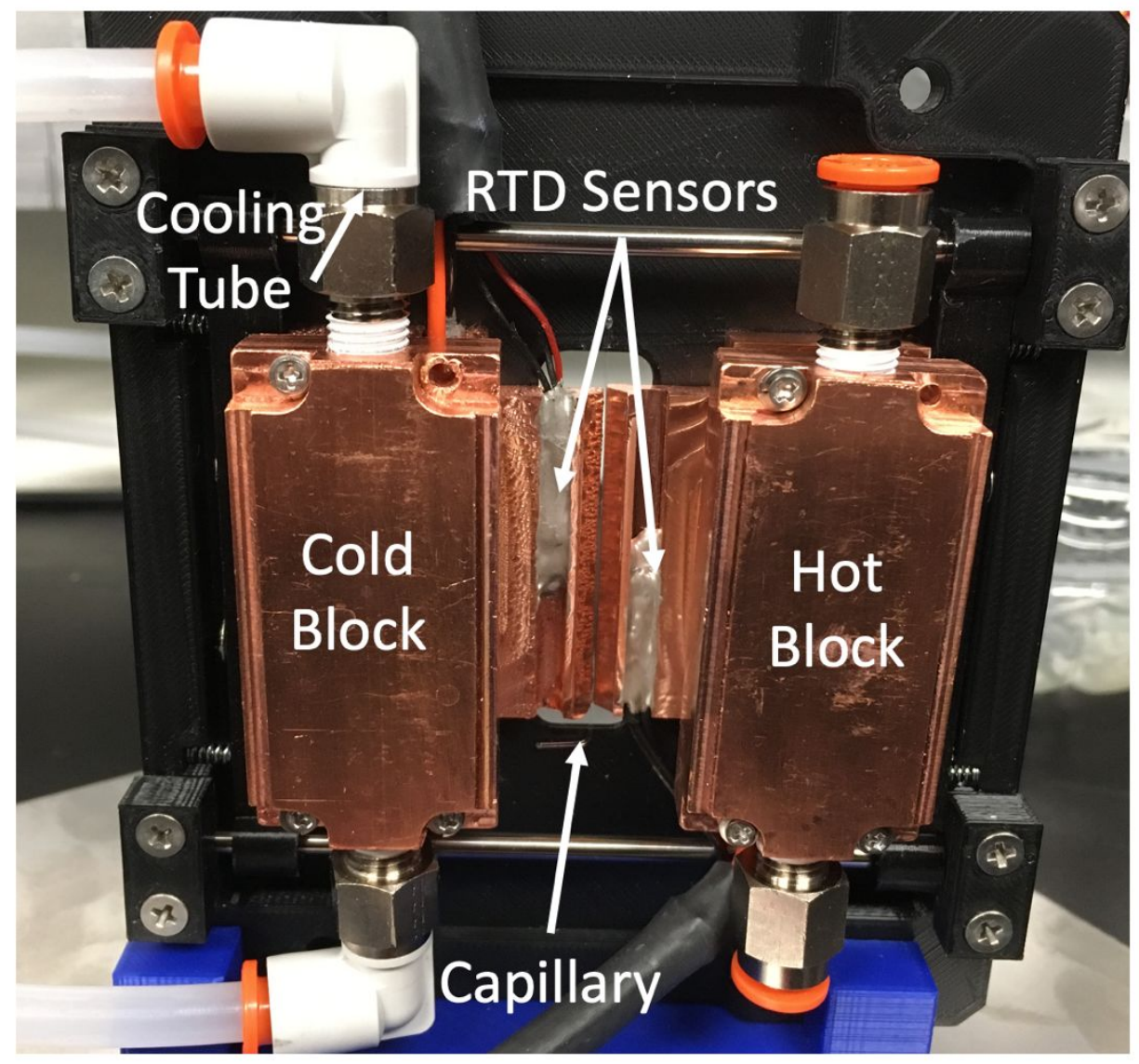

Figure S1. Annotated photograph of the active elements of the Thermogravitational Clusius-Dickel Micro-Device. All components are held in place by a 3D-printed polylacticacid frame (black) and base (blue). The spring-loaded sliders are visible on either side of the frame.

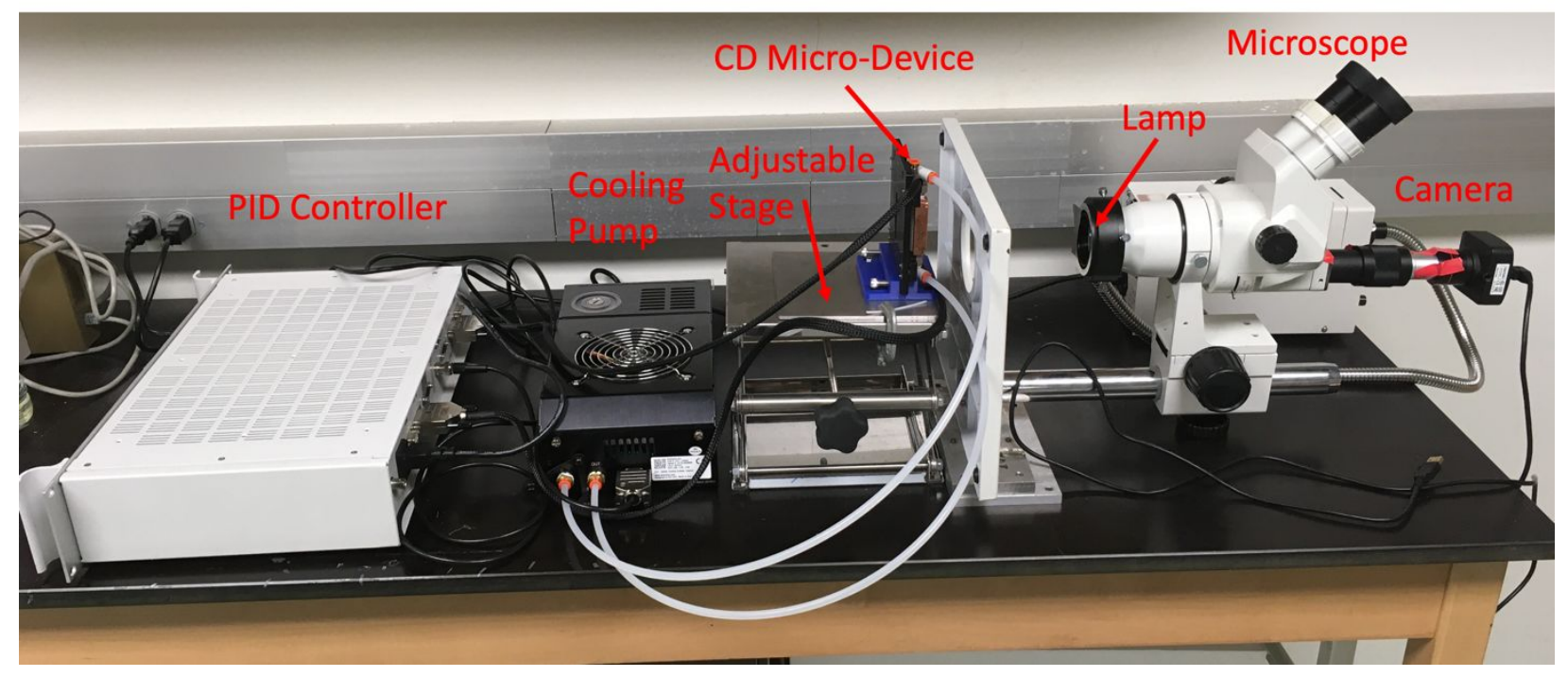


Figure S2. Annotated photograph of an early iteration of the entire experimental setup as described in the main text. The microscope lens was eventually moved closer to the micro-device sample and mounted on a 3D-printed stand to minimize tilting.

Simulation. In order to confirm the validity of the theoretical model of the thermogravitational Clusius-Dickel separation, a simplified simulation of colloids inside the micro-device was developed using the Python library Pygame. The simulation tracks the position of randomly diffusing particles inside a confined two-dimensional space. Every computation step of the simulation, each particle moves a set distance in a random angular direction, unless pushed back by one of the walls of the container. A snapshot image of the simulation, with the particles colored green, is shown in Figure S3A.

To simulate a stable natural convection current, an additional vector was applied to each particle based on its current position in the container. In the central $\Delta \mathrm{T}$ region, colored red and blue and marked by arrows, the particle was moved up or down by a convection vector depending on whether it is closer to the "hot" or "cold" wall. The strength of this movement was proportional to how close the particle is to the center of the upward or downward current. Meanwhile, in the purple transition region, the additional convection vector transported particles in a semicircular arc, moving them clockwise from one current into the other. No convection vector was applied in the white "collection" region These convection patterns were derived from a separate simulation of stable natural convection in a narrow channel geometry, of which Figure S3B shows the flow profile. This convection simulation was performed by using the COMSOL finite element analysis software to solve the Rayleigh-Bérnard buoyancy approximation and heat transfer equation at steady-state for a long, thin container with walls at constant temperature and an insulating top and bottom. 

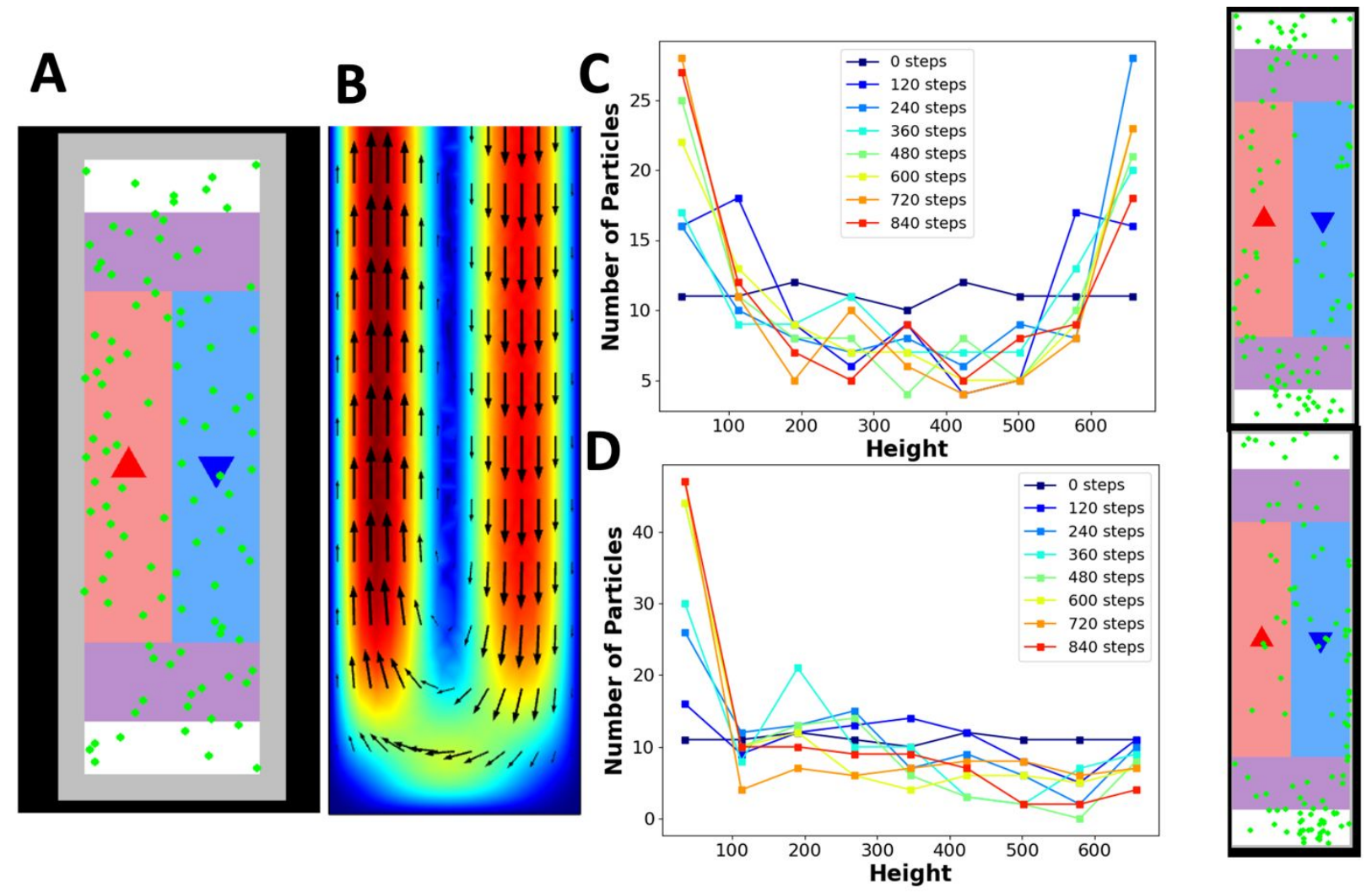

Figure S3: Simulation of thermogravitational separation. (A) Initial snapshot of the Python simulation. (B) Arrow surface representation of the flow profile near the transition zone obtained by COMSOL simulation of natural convection. Warmer colors represent larger magnitudes of convective flow. (C and D) Number of particles as a function of height for increasing simulation steps, adjacent to a snapshot of the simulation end state, for diffusion + Convection (C) and diffusion + convection + thermophoresis (D).

By recording the position of each particle after a certain number of steps within the Python simulation, a time evolution of the vertical particle distribution within the container was obtained. Figure S3C shows that diffusion and convection alone are sufficient to concentrate the particle in the upper and lower diffusion regions, away from the high-velocity $\Delta \mathrm{T}$ region. With the introduction of thermophoresis into the simulation, represented by a third vector component that moves particles horizontally away from the left "hot" wall, the distribution became biased to 
the lower diffusion region, as seen in Figure S3D. This is the same qualitative result obtained by more rigorous finite-element simulation of Clusius-Dickel separation by Dieter Braun (30). Overall, the Python simulation demonstrates that thermally biased Brownian motion is sufficient to explain the macro-scale effects of the thermogravitational technique.

Video Frames. Figures S4 and S5 feature a series of still images from video of the latex colloids moving in the micro-device during thermogravitational separation. The time difference between each sequential adjacent frame is approximately two seconds. In each frame, the right side of the capillary is the cold wall and the left side is the hot wall. From the image series, the antiparallel movement of the colloids, down on the right side and up on the left side, can be easily visualized. y coordinates are given in reference to the bottom of the $\Delta \mathrm{T}$ zone at the lower edge of the heating blocks. 


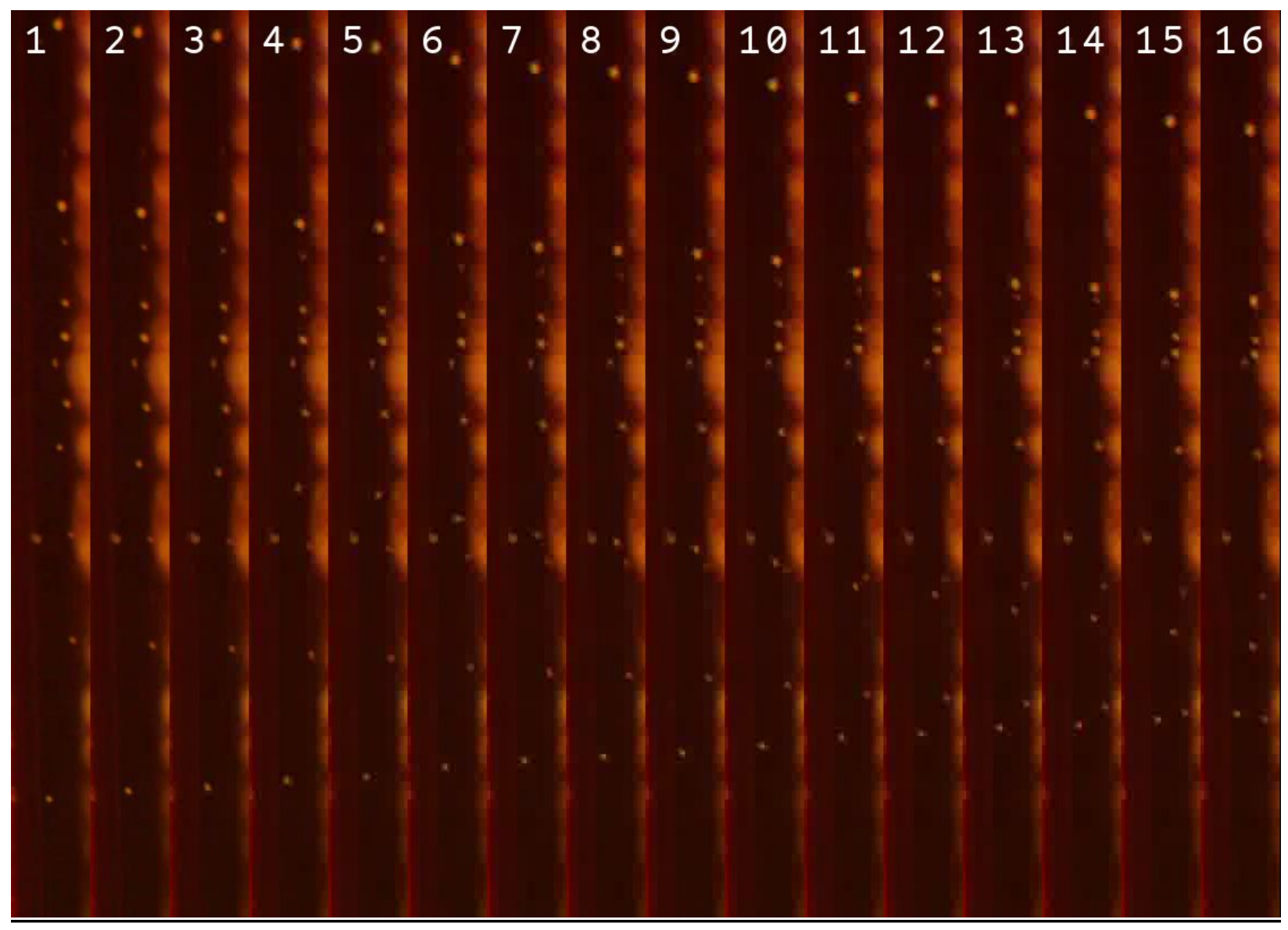

Figure S4. Video frames of colloid movement at $\mathrm{y}=22-26 \mathrm{~mm}$, after 20 minutes with $\Delta \mathrm{T}=50 \mathrm{~K}$. 


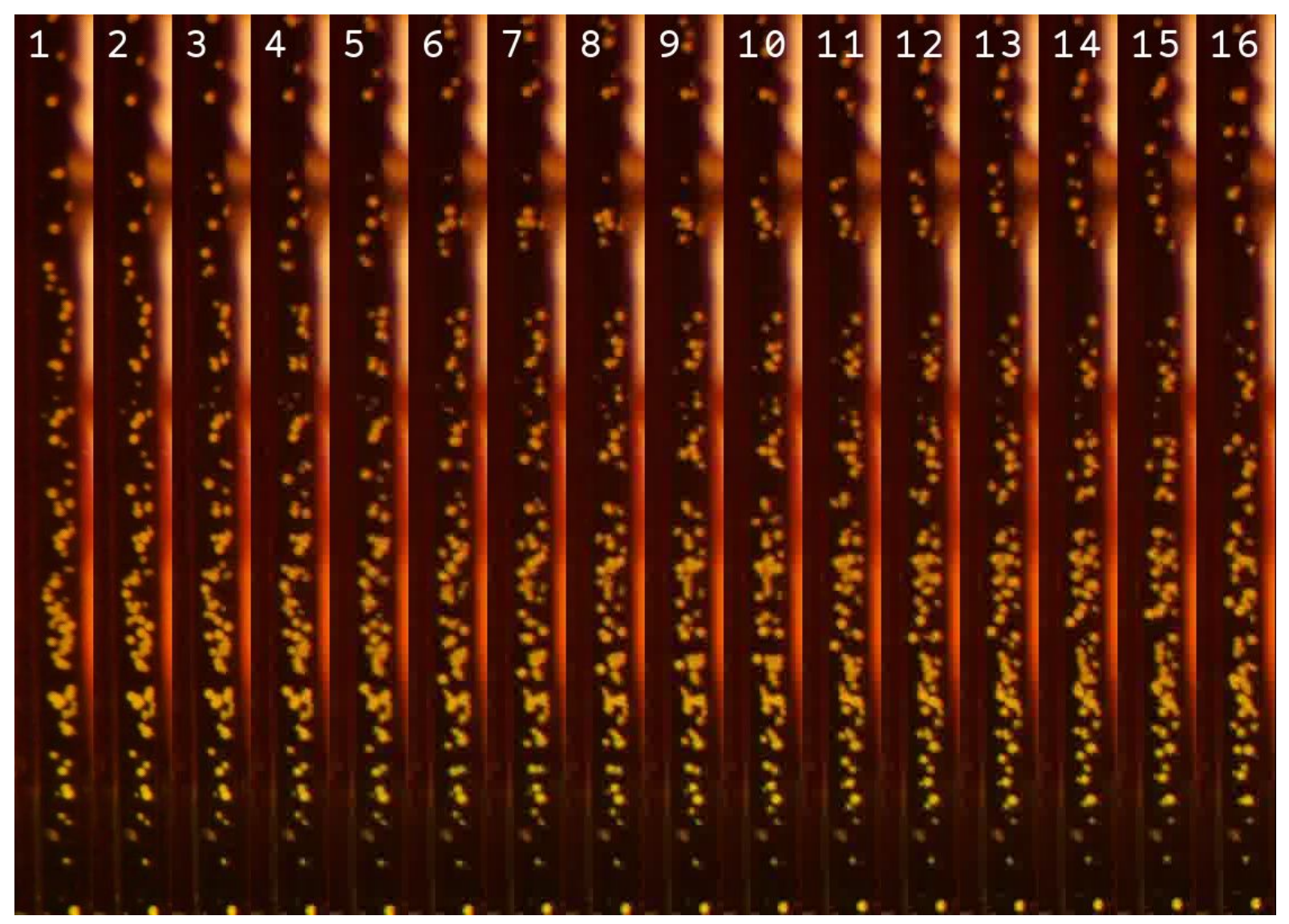

Figure S5. Video frames of colloid movement at $\mathrm{y}=0-4 \mathrm{~mm}$, after 20 minutes with $\Delta \mathrm{T}=50 \mathrm{~K}$.

Estimation of Error. The table below organizes the three main sources of experimental variance for the polyethylene colloid measurements. Through a method involving interpolation and the equation for the bottom-top concentration ratio from reference 27 (equation S1), the propagation of error to its potential effect on the average height of colloids in the column (figure $6, \mathrm{~B}$ and $\mathrm{C}$ ) was calculated. In this equation, $\mathrm{R}$ is the aspect ratio of the cell, approximately equal to 190 for our experiments.

$$
\frac{c_{\text {bottom }}}{c_{\text {top }}}=\exp \left(R \bullet \Delta T \cdot S_{T}\right)
$$

For the variance in particle diameter, it was assumed $\mathrm{S}_{\mathrm{T}}$ is directly proportional to particle radius $\left(\mathrm{S}_{\mathrm{T}}=\mathrm{D}_{\mathrm{T}} / \mathrm{D}, \mathrm{D} \propto 1 / \mathrm{r}\right)$. The variance in $\mathrm{R}$ and particle diameter was obtained from manufacturer variation, variance in $\Delta \mathrm{T}$ derives from the typical range in room temperature, which two walls of 
the capillary are exposed to. The root-mean-squared sum of these errors is the error for each bar pair in figure 6, B and C, added to the baseline "convection drift" error of $\pm 2.9 \mathrm{~mm}$. If this error could not be calculated, only the baseline "convection drift" error was used.

\begin{tabular}{|l|l|l|l|}
\hline $\begin{array}{l}\text { Source of } \\
\text { Error }\end{array}$ & $\begin{array}{l}\text { Estimated } \\
\text { Source } \\
\text { Variance }\end{array}$ & $\begin{array}{l}\text { Mean } \\
\text { Concentration } \\
\text { Ratio Variance }\end{array}$ & $\begin{array}{l}\text { Mean Average Particle } \\
\text { Height Variance }\end{array}$ \\
\hline $\mathrm{R}$ & $\pm 11 \%$ & \pm 0.55 & $\pm 0.35 \mathrm{~mm}$ \\
\hline$\Delta \mathrm{T}$ & $\pm 3 \%$ & \pm 0.2 & $\pm 0.13 \mathrm{~mm}$ \\
\hline $\mathrm{S}_{\mathrm{T}}$ & $\pm 8.4 \%$ & \pm 0.45 & $\pm 0.28 \mathrm{~mm}$ \\
\hline RMS Sum & & \pm 0.75 & \pm 0.47 \\
\hline
\end{tabular}

